\title{
An extremely metal-deficient globular cluster in the Andromeda Galaxy
}

\author{
Søren S. Larsen ${ }^{1 *}$, Aaron J. Romanowsky ${ }^{2,3}$, Jean P. Brodie ${ }^{3,4}$, Asher Wasserman ${ }^{3}$ \\ ${ }^{1}$ Department of Astrophysics/Institute of Mathematics, Astrophysics and Particle Physics, Radboud University, 6500 GL Nijmegen, Netherlands. ${ }^{2}$ Department of \\ Physics and Astronomy, San José State University, San Jose, CA 95192, USA. ${ }^{3}$ University of California Observatories, University of California, Santa Cruz, CA \\ 95064, USA. ${ }^{4}$ Centre for Astrophysics and Supercomputing, Swinburne University of Technology, Hawthorn, VIC 3122, Australia. \\ *Corresponding author. Email: s.larsen@astro.ru.nl \\ Globular clusters (GCs) are dense, gravitationally bound systems of thousands to millions of stars. They \\ are preferentially associated with the oldest components of galaxies, so measurements of their \\ composition can constrain the build-up of chemical elements in galaxies during the early Universe. We \\ report a massive GC in the Andromeda Galaxy (M31), RBC EXT8, that is extremely depleted in heavy \\ elements. Its iron abundance is about $\mathbf{8 0 0}$ times lower than that of the Sun, and about three times lower \\ than the most iron-poor GCs previously known. It is also strongly depleted in magnesium. These \\ measurements challenge the notion of a metallicity floor for GCs and theoretical expectations that \\ massive GCs could not have formed at such low metallicities.
}

Globular clusters (GCs) are roughly spherical agglomerations of thousands to millions of stars, bound by their mutual gravity; their central densities can exceed $10^{6}$ solar masses per cubic parsec $\left(M_{\odot} \mathrm{pc}^{-3}\right)(1)$. GCs formed early in the history of the Universe and therefore record the early stages of galaxy formation and evolution. The nearest neighboring spiral galaxy, the Andromeda Galaxy, also known as Messier 31 (M31), has a system of GCs that align spatially and kinematically with stars in the outer parts of the galaxy. The GCs in the outer parts of M31 appear to belong to at least two kinematically distinct subsystems that were accreted separately (2).

The GC systems in most galaxies are dominated by clusters with low abundances of elements heavier than hydrogen and helium ("metals"), relative to the composition of the Sun. However, there appears to be a deficit of GCs at the very lowest metal abundances ("metallicities") (3). The most metal-poor GCs in the Milky Way have iron abundances of $[\mathrm{Fe} / \mathrm{H}] \approx-2.5(4)$, where square brackets denote the abundance ratios of the elements, relative to the solar photospheric composition, on a logarithmic scale. The number of iron atoms per hydrogen atom in the most metal-poor GCs is thus about 300 times lower than in the Sun. The notion of a metallicity floor for GCs at $[\mathrm{Fe} / \mathrm{H}]=-2.5$ is supported by observations of GCs in several external galaxies (5), and various explanations have been suggested. The correlation between mass and metallicity for galaxies in the early Universe might set a minimum metallicity for formation of
GCs that are sufficiently massive to survive until the present day, or the formation of massive GCs could be suppressed at low metallicities due to inefficient gas cooling (3, 5-7). A metallicity floor for GCs would thus have implications for cluster- and star formation and for the build-up of metals in galaxies during the early Universe.

Because the metallicity distributions of both GCs and individual stars decline steeply toward low metallicities and are poorly constrained, it remains unclear how statistically significant the proposed metallicity floor is. In M31, three clusters with metallicities that may fall in the range $-2.8<$ $[\mathrm{Fe} / \mathrm{H}]<-2.5$ are known $(8)$, but the uncertainties are large (0.3-0.4 dex) and the metallicities may lie well above the floor. Similarly, three GCs in the Sombrero Galaxy may have metallicities below $[\mathrm{Fe} / \mathrm{H}]=-2.5(9)$, but the uncertainties on the spectroscopic measurements are large and the red colors of these clusters suggest higher metallicities.

We investigate the globular cluster RBC EXT8 (hereafter EXT8) in M31, located at right ascension $00^{h} 53^{m} 14^{s} .53$, declination $+41^{\circ} 33^{\prime} 24^{\prime \prime} .5$ (J2000 equinox) according to the Revised Bologna Catalogue (10). Kinematic analysis (2) has shown that EXT8 belongs to the smoothly distributed component of the M31 halo, and lies at a projected distance of $27 \mathrm{kpc}$ from the galaxy center. Figure 1 shows a colormagnitude diagram for GCs in M31 (11). With an apparent magnitude in the $g$-band of $g=15.87$, EXT8 is among the brighter GCs in M31. Its integrated light color with respect to the $u$-band ( $u-g=1.11)$ is less red than most of the other 
GCs, suggesting a low metallicity. Previous low-resolution spectroscopy yielded an age $\geq 8 \mathrm{Gyr}$ and $[\mathrm{Fe} / \mathrm{H}]$ between -2.8 and $-2.0(12,13)$.

We obtained a spectrum of the integrated light of EXT8 with the High-Resolution Echelle Spectrometer (HIRES) (14) on the Keck I telescope on 25 Oct 2019. Given EXT8's brightness and compact size, a total integration time of $2400 \mathrm{~s}$ was sufficient to obtain a signal-to-noise ratio of about 200 per $\AA$ near the $\mathrm{Mg}$ I triplet at $5170 \AA$. We used a slit width of 1.15" which gave a nominal spectral resolving power $R \equiv \lambda / \Delta \lambda \approx 37000$, where $\lambda$ is the wavelength and $\Delta \lambda$ the width of a spectral resolution element. The observations covered a spectral range of 3840-8060 A.

Figure 2 shows the $H \beta$ lines in the spectra of EXT8 and Messier 15 (M15) for comparison, the latter being one of the most metal-poor GCs in the Milky Way (15). The spectrum of M15 was obtained with the Ultraviolet and Visual Echelle Spectrograph (UVES) on the Very Large Telescope and has a spectral resolving power similar to that of the EXT8 spectrum (16). M15's velocity dispersion $\left(\sigma=12.9 \pm 0.3 \mathrm{~km} \mathrm{~s}^{-1}\right)$ is similar to that of EXT8 $\left(\sigma=13.3 \pm 0.8 \mathrm{~km} \mathrm{~s}^{-1}\right)$, allowing direct comparison. Absorption in the $\mathrm{H} \beta$ line becomes stronger at younger ages and can be used as an age indicator in the spectra of GCs (17). While the blue color of EXT8 could, in principle, be caused by a younger age, Fig. 2 shows no discernible difference in the strengths or shapes of the $\mathrm{H}$ $\beta$ lines in the two spectra, indicating that EXT8 is similarly old, so must be a metal-poor GC.

Figure 3 shows two metallicity-sensitive features. Figure $3 \mathrm{~A}$ shows the $\mathrm{Fe}$ I feature near $4957 \AA$ (actually a blend of several Fe I lines, of which the two strongest are marked), which is much weaker in the spectrum of EXT8 than in M15. Figure 3B shows two of the three lines of the Mg I triplet (Fraunhofer's $b$ feature) at $5167 \AA$ and $5173 \AA$. The third line, at $5184 \AA$, falls in the gap between the two detectors of UVES, but is included in the HIRES spectrum. The Mg I lines, as well as other lines visible in this region of the spectra, are much weaker in the EXT8 spectrum.

To quantify these results, we analyzed the EXT8 spectrum using a spectral fitting technique used in previous studies of extragalactic GCs $(16,18)$. Figure 3 shows the bestfitting model spectrum for M15 (16), with an iron abundance of $[\mathrm{Fe} / \mathrm{H}]=-2.39 \pm 0.02$. This model spectrum is based on a color-magnitude diagram (CMD) of M15 (19). We do not have spatially resolved data to empirically build a CMD for EXT8, so substituted it with stellar models (20) with a metal fraction chosen to self-consistently match that derived from the spectral modeling. We found an iron abundance of $[\mathrm{Fe} / \mathrm{H}]=-2.91 \pm 0.04$ for EXT8 from model fitting of the wavelength range 4400-6200 $\AA$ (19). These model spectra are also shown in Fig. 3. We tested the assumptions required for the input CMD and found that they do not sub- stantially affect this measurement (19). We conclude that EXT8 is about 0.5 dex more metal-poor than the value of $[\mathrm{Fe} / \mathrm{H}]=-2.39$ found for M15. The metallicity of EXT8 lies well below the metallicity floor suggested by previous studies.

At the signal-to-noise ratio of our EXT8 spectrum, most spectral features are weak because of the low metallicity. Nevertheless, our model fitting yielded abundances for several additional elements. From the $\mathrm{Mg}$ I $b$ lines we found a low magnesium abundance of $[\mathrm{Mg} / \mathrm{Fe}]=-0.35 \pm 0.05$. Magnesium is thus even more strongly depleted than iron, relative to the Sun. The other $\mathrm{Mg}$ lines that are typically measured in integrated-light GC spectra are very weak (19), but are generally consistent with the low magnesium abundance inferred from the $b$ lines: $[\mathrm{Mg} / \mathrm{Fe}]=-0.16_{-0.58}^{+0.27}$ (using Mg I $4571 \AA \AA),[\mathrm{Mg} / \mathrm{Fe}]=-0.96_{-\infty}^{+0.65}(\mathrm{Mg}$ I $4703 \AA$ ), and $[\mathrm{Mg} / \mathrm{Fe}]=-0.35 \pm 0.25(\mathrm{Mg}$ I $5528 \AA)$. The consistency between the magnesium abundances measured from the $b$ triplet and from the weaker lines is supported by prior analysis of M15, where the weaker lines yielded $[\mathrm{Mg} / \mathrm{Fe}]=+0.18$ \pm 0.06 (16). From our model fitting of M15, we find an almost identical abundance of $[\mathrm{Mg} / \mathrm{Fe}]=+0.17 \pm 0.02$ (using the two $\mathrm{Mg} b$ lines included in the UVES spectrum). Magnesium is among the elements thought to be produced via the $\alpha$ process of nucleosynthesis (21). For other $\alpha$-elements, we find average abundance ratios of $[\mathrm{Si} / \mathrm{Fe}]=+0.65 \pm 0.31$, $[\mathrm{Ca} / \mathrm{Fe}]=+0.35 \pm 0.07$, and $[\mathrm{Ti} / \mathrm{Fe}]=+0.19 \pm 0.06$ for EXT8 (19). Relative to iron, these elements are thus on average enhanced by roughly a factor of two compared to the composition of the solar photosphere.

The enhanced abundances of silicon, calcium, and titanium are typical for metal-poor, old populations, which is usually attributed to enrichment of $\alpha$-elements dominated by core-collapse supernovae (22). However, the very low magnesium abundance is not easily explained within this framework. It may be related to the phenomenon of multiple stellar populations in GCs, of which the outer halo cluster NGC 2419 is one of the most extreme cases in the Milky Way $(23,24)$. In NGC 2419, some individual stars have magnesium abundances as low as $[\mathrm{Mg} / \mathrm{Fe}]=-1$, although more typical values for the Mg-depleted stars in NGC 2419 (which constitute about $40 \%$ of the stars in this cluster) are $[\mathrm{Mg} / \mathrm{Fe}] \approx-0.5$. The distribution of $[\mathrm{Mg} / \mathrm{Fe}]$ values within EXT8 is not constrained by our measurements, but the $[\mathrm{Mg} / \mathrm{Fe}]$ value measured from the integrated light can be reproduced if the cluster contains two populations with $[\mathrm{Mg} / \mathrm{Fe}] \approx-1.0$ and $[\mathrm{Mg} / \mathrm{Fe}] \approx+0.3$ that each account for about half of the stars (19). In this case, a larger difference between two populations is required in EXT8 than in NGC 2419.

Further evidence for multiple populations in EXT8 
comes from the abundance of sodium. From the Na I resonance doublet at 5890, $5896 \AA$ (Fraunhofer's $D$ feature) we find sodium to be enhanced relative to scaled-solar composition, $[\mathrm{Na} / \mathrm{Fe}]=+0.23 \pm 0.07$, as is commonly observed in GCs (25). However, the $D$ lines may be contaminated by absorption from interstellar gas along the line-of-sight toward EXT8. Other $\mathrm{Na}$ I lines that are immune to this effect are very weak in the spectrum of EXT8, but from the doublet at $5683,5688 \AA$ we measure $[\mathrm{Na} / \mathrm{Fe}]=+0.26_{-\infty}^{+0.32}$, which is consistent with the value inferred from the $D$ lines.

In NGC 2419, the Mg-poor stars are also enriched in $\mathrm{K}$, reaching $[\mathrm{K} / \mathrm{Fe}] \approx+1.5(23,24)$. For EXT8 we measure $[\mathrm{K} / \mathrm{Fe}]=+0.67 \pm 0.15$, but this value may require correction downward by about 0.3 dex to account for our assumption of local thermodynamic equilibrium in the spectral modeling (26). This would then make the $[\mathrm{K} / \mathrm{Fe}]$ ratio in EXT8 similar to that observed in metal-poor halo stars in the Milky Way and in M15 (27). Hence, we find no evidence of a K-enriched population in EXT8.

From the model fitting, we also determined the velocity broadening of the observed spectrum. The line-of-sight velocity dispersion, corrected for instrumental broadening, is $\sigma=13.3 \pm 0.8 \mathrm{~km} \mathrm{~s}^{-1}$. The half-light radius of EXT8 is $2.8 \mathrm{pc}$, leading to an estimated dynamical mass of $M_{\mathrm{dyn}}=(1.14 \pm$ $0.16) \times 10^{6} M_{\odot}(19)$. For an absolute magnitude in the $V$ band of $M_{V}=-9.28$ (28), the corresponding mass-to-light ratio in solar units is $M_{\mathrm{dyn}} / L_{V}=2.6 M_{\odot} / L_{\odot, V}$, where $L_{V}$ and $L_{\odot, V}$ are the luminosities of EXT8 and the Sun in the $V$-band. EXT8 thus extends the trend for metal-poor GCs to have high $M / L$ values (29). A lower $M / L$ would be expected for a younger age, so the measured value is consistent with EXT8 being an old, metal-poor system.

Figure 4 shows a comparison of EXT8 with previous integrated-light spectroscopy of Galactic and extragalactic GCs $(16,18)$, along with literature data for individual stars in Galactic GCs and individual stars $(30,31)$. EXT8 is an outlier in a $[\mathrm{Mg} / \mathrm{Fe}]$ vs. $[\mathrm{Fe} / \mathrm{H}]$ plot (Fig. $4 \mathrm{~A}$ ), being more metalpoor than other GCs and more magnesium poor than individual stars with similarly low iron abundances. The GCs have a larger spread in $[\mathrm{Mg} / \mathrm{Fe}]$ than the individual stars, with scatter toward lower magnesium abundances. This has previously been interpreted as a signature of multiple populations $(32,33)$. When excluding magnesium, Fig. 4B shows that EXT8 has an $[\alpha / \mathrm{Fe}]$ ratio (here defined as the average of $[\mathrm{Ca} / \mathrm{Fe}]$ and $[\mathrm{Ti} / \mathrm{Fe}])$ that overlaps with those seen in individual metal-poor stars and in other GCs.

Within the standard paradigm of hierarchical galaxy assembly, metal-poor GCs are expected to have formed in the early Universe in low-mass galaxies that merged to form larger galaxies $(6,7,34)$. The correlation between the mass and metallicity of galaxies therefore imprints a maximum mass for a GC that could form with a given metallicity. At $[\mathrm{Fe} / \mathrm{H}]=-2.9$, the maximum mass is expected to be about $10^{5} M_{\odot}(6,7)$. The existence of a possible remnant of a disrupted GC in the Milky Way with $[\mathrm{Fe} / \mathrm{H}]=-2.7$ and an estimated mass below $10^{5} M_{\odot}$ (35) is consistent with this notion. However, we expect clusters as massive and metalpoor as EXT8 to be extremely rare. In a simulation containing 10,553 GCs with masses greater than $10^{5} M_{\odot}$, only three $(\sim 0.03 \%)$ had $[\mathrm{Fe} / \mathrm{H}]<-2.5$ and masses above $10^{6} M_{\odot}(36)$, where we have converted the total metal fractions (36) to $[\mathrm{Fe} / \mathrm{H}]$ values (19). If half of the $400-500 \mathrm{GCs}$ in M31 (10) have masses greater than $10^{5} M_{\odot}$, this would correspond to a probability of $6-7 \%$ of finding a single GC as massive and metal-poor as EXT8.

\section{REFERENCES AND NOTES}

1. H. Baumgardt, M. Hilker, A catalogue of masses, structural parameters, and velocity dispersion profiles of 112 Milky Way globular clusters. Mon. Not. $R$. Astron. Soc. 478, 1520-1557 (2018). doi:10.1093/mnras/sty1057

2. D. Mackey, G. F. Lewis, B. J. Brewer, A. M. N. Ferguson, J. Veljanoski, A. P. Huxor, M. L. M. Collins, P. Côté, R. A. Ibata, M. J. Irwin, N. Martin, A. W. McConnachie, J. Peñarrubia, N. Tanvir, Z. Wan, Two major accretion epochs in M31 from two distinct populations of globular clusters. Nature 574, 69-71 (2019). doi:10.1038/s41586-019-1597-1 Medline

3. B. W. Carney, J. B. Laird, D. W. Latham, L. A. Aguilar, A Survey of Proper Motion Stars. XIII. The Halo Population. Astron. J. 112, 668 (1996). doi:10.1086/118042

4. J. D. Simpson, The most metal-poor Galactic globular cluster: The first spectroscopic observations of ESO280-SC06. Mon. Not. R. Astron. Soc. 477, 4565-4576 (2018). doi:10.1093/mnras/sty847

5. M. A. Beasley, R. Leaman, C. Gallart, S. S. Larsen, G. Battaglia, M. Monelli, M. H. Pedreros, An old, metal-poor globular cluster in Sextans A and the metallicity floor of globular cluster systems. Mon. Not. R. Astron. Soc. 487, 1986-1993 (2019). doi:10.1093/mnras/stz1349

6. N. Choksi, O. Y. Gnedin, H. Li, Formation of globular cluster systems: From dwarf galaxies to giants. Mon. Not. R. Astron. Soc. 480, 2343-2356 (2018). doi:10.1093/mnras/sty1952

7. J. M. D. Kruijssen, The minimum metallicity of globular clusters and its physical origin-implications for the galaxy mass-metallicity relation and observations of proto-globular clusters at high redshift. Mon. Not. R. Astron. Soc. 486, L20-L25 (2019). doi:10.1093/mnras//slz052

8. N. Caldwell, R. Schiavon, H. Morrison, J. A. Rose, P. Harding, Star clusters in M31. II. Old cluster metallicities and ages from hectospec data. Astron. J. 141, 61 (2011). doi:10.1088/0004-6256/141/2/61

9. A. Alves-Brito, G. K. T. Hau, D. A. Forbes, L. R. Spitler, J. Strader, J. P. Brodie, K. L. Rhode, Spectra of globular clusters in the Sombrero galaxy: Evidence for spectroscopic metallicity bimodality. Mon. Not. R. Astron. Soc. 417, 1823-1838 (2011). doi:10.1111/j.1365-2966.2011.19368.x

10. S. Galleti, L. Federici, M. Bellazzini, F. F. Pecci, S. Macrina, 2MASS NIR photometry for 693 candidate globular clusters in M 31 and the Revised Bologna Catalogue. Astron. Astrophys. 416, 917-924 (2004). doi:10.1051/00046361:20035632

11. M. B. Peacock, T. J. Maccarone, C. Knigge, A. Kundu, C. Z. Waters, S. E. Zepf, D. R. Zurek, The M31 globular cluster system: Ugriz and K-band photometry and structural parameters. Mon. Not. R. Astron. Soc. 402, 803-818 (2010). doi:10.1111/j.1365-2966.2009.15952.x

12. Z. Fan, Y.-F. Huang, J.-Z. Li, X. Zhou, J. Ma, H. Wu, T.-M. Zhang, Y.-H. Zhao, 
Spectroscopic study of globular clusters in the halo of M31 with the Xinglong 2.16 m telescope. Res. Astron. Astrophys. 11, 1298-1310 (2011). doi:10.1088/16744527/11/11/005

13. B. Chen, X. Liu, M. Xiang, H. Yuan, Y. Huang, J. Shi, Z. Fan, Z. Huo, C. Wang, J. Ren, Z. Tian, H. Zhang, G. Liu, Z. Cao, Y. Zhang, Y. Hou, Y. Wang, The Lamost spectroscopic survey of star clusters in M31. Il. Metallicities, ages, and masses. Astron. J. 152, 45 (2016). doi:10.3847/0004-6256/152/2/45

14. S. S. Vogt et al., HIRES: The high-resolution Echelle spectrometer on the Keck $10-$ m Telescope. Proc. SPIE 2198, 362 (1994).

15. W. E. Harris, A Catalog of Parameters for Globular Clusters in the Milky Way. Astron. J. 112, 1487 (1996). doi:10.1086/118116

16. S. S. Larsen, J. P. Brodie, J. Strader, Detailed abundances from integrated-light spectroscopy: Milky Way globular clusters. Astron. Astrophys. 601, A96 (2017). doi:10.1051/0004-6361/201630130

17. G. Worthey, Comprehensive stellar population models and the disentanglement of age and metallicity effects. Astrophys. J. Suppl. Ser. 95, 107 (1994). doi:10.1086/192096

18. S. S. Larsen, J. P. Brodie, A. Wasserman, J. Strader, Detailed abundance analysis of globular clusters in the Local Group. Astron. Astrophys. 613, A56 (2018). doi:10.1051/0004-6361/201731909

19. See supplementary materials.

20. J. Choi, A. Dotter, C. Conroy, M. Cantiello, B. Paxton, B. D. Johnson, MESA isochrones and stellar tracks (MIST). I. Solar-scaled models. Astrophys. J. 823, 102 (2016). doi:10.3847/0004-637X/823/2/102

21. E. Burbidge, G. Burbidge, W. Fowler, F. Hoyle, Synthesis of the Elements in Stars. Rev. Mod. Phys. 29, 547-650 (1957). doi:10.1103/RevModPhys.29.547

22. B. M. Tinsley, Stellar lifetimes and abundance ratios in chemical evolution. Astrophys. J. 229, 1046 (1979). doi:10.1086/157039

23. J. G. Cohen, E. N. Kirby, The bizarre chemical inventory of NGC 2419, an extreme outer halo globular cluster. Astrophys. J. 760, 86 (2012). doi:10.1088/0004$637 \times / 760 / 1 / 86$

24. A. Mucciarelli, M. Bellazzini, R. Ibata, T. Merle, S. C. Chapman, E. Dalessandro, A. Sollima, News from the Galactic suburbia: The chemical composition of the remote globular cluster NGC 2419. Mon. Not. R. Astron. Soc. 426, 2889-2900 (2012). doi:10.1111/j.1365-2966.2012.21847.x

25. E. Carretta, A. Bragaglia, R. G. Gratton, S. Lucatello, G. Catanzaro, F. Leone, M. Bellazzini, R. Claudi, V. D'Orazi, Y. Momany, S. Ortolani, E. Pancino, G. Piotto, A. Recio-Blanco, E. Sabbi, Na-O anticorrelation and HB. Astron. Astrophys. 505, 117-138 (2009). doi:10.1051/0004-6361/200912096

26. Y. Takeda, G. Zhao, Y.-Q. Chen, H.-M. Qiu, M. Takada-Hidai, On the Abundance of Potassium in Metal-Poor Stars. Publ. Astron. Soc. Jpn. 54, 275-284 (2002). doi:10.1093/pasj/54.2.275

27. Y. Takeda, H. Kaneko, N. Matsumoto, S. Oshino, H. Ito, T. Shibuya, Potassium Abundances in Red Giants of Mildly to Very Metal-Poor Globular Clusters. Publ. Astron. Soc. Jpn. 61, 563-576 (2009). doi:10.1093/pasj/61.3.563

28. A. P. Huxor, A. D. Mackey, A. M. N. Ferguson, M. J. Irwin, N. F. Martin, N. R. Tanvir, J. Veljanoski, A. McConnachie, C. K. Fishlock, R. Ibata, G. F. Lewis, The outer halo globular cluster system of M31-I. The final PAndAS catalogue. Mon. Not. R. Astron. Soc. 442, 2165-2187 (2014). doi:10.1093/mnras/stu771

29. J. Strader, N. Caldwell, A. C. Seth, Star clusters in M31. V. Internal dynamical trends: Some troublesome, some reassuring. Astron. J. 142, 8 (2011). doi:10.1088/0004-6256/142/1/8

30. B. J. Pritzl, K. A. Venn, M. Irwin, A Comparison of Elemental Abundance Ratios in Globular Clusters, Field Stars, and Dwarf Spheroidal Galaxies. Astron. J. 130, 2140-2165 (2005). doi:10.1086/432911

31. K. A. Venn, M. Irwin, M. D. Shetrone, C. A. Tout, V. Hill, E. Tolstoy, Stellar Chemical Signatures and Hierarchical Galaxy Formation. Astron. J. 128, 1177-1195 (2004). doi:10.1086/422734

32. J. E. Colucci, R. A. Bernstein, S. Cameron, A. McWilliam, J. G. Cohen, M31 globular cluster abundances from high-resolution, integrated-light spectroscopy. Astrophys. J. 704, 385-414 (2009). doi:10.1088/0004637X/704/1/385

33. C. M. Sakari, M. Shetrone, K. Venn, A. McWilliam, A. Dotter, Spectrum syntheses of high-resolution integrated light spectra of Galactic globular clusters. Mon. Not. R. Astron. Soc. 434, 358-386 (2013). doi:10.1093/mnras/stt1026

34. W. E. Harris, B. C. Whitmore, D. Karakla, W. Okoń, W. A. Baum, D. A. Hanes, J. J. Kavelaars, Globular Cluster Systems in Brightest Cluster Galaxies: Bimodal Metallicity Distributions and the Nature of the High-Luminosity Clusters. Astrophys. J. 636, 90-114 (2006). doi:10.1086/498058

35. Z. Wan, G. F. Lewis, T. S. Li, J. D. Simpson, S. L. Martell, D. B. Zucker, J. R. Mould, D. Erkal, A. B. Pace, D. Mackey, A. P. Ji, S. E. Koposov, K. Kuehn, N. Shipp, E. Balbinot, J. Bland-Hawthorn, A. R. Casey, G. S. Da Costa, P. Kafle, S. Sharma, G. M. De Silva, The tidal remnant of an unusually metal-poor globular cluster. Nature 583, 768-770 (2020). doi:10.1038/s41586-020-2483-6 Medline

36. C. Usher, J. Pfeffer, N. Bastian, J. M. D. Kruijssen, R. A. Crain, M. Reina-Campos, The origin of the 'blue tilt' of globular cluster populations in the E-MOSAICS simulations. Mon. Not. R. Astron. Soc. 480, 3279-3301 (2018). doi:10.1093/mnras/sty1895

37. S. Larsen, ISPy3: Integrated-light Spectroscopy for Python3. Zenodo, DOI 10.5281/zenodo.4036092 (2020)

38. S. S. Larsen, Young massive star clusters in nearby galaxies. Astron. Astrophys. Suppl. Ser. 139, 393-415 (1999). doi:10.1051/aas:1999509

39. T. Barlow, MAKEE User Guide and Technical Documentation (2019); https://sites.astro.caltech.edu/ tb/makee/.

40. P. Kroupa, On the variation of the initial mass function. Mon. Not. R. Astron. Soc. 322, 231-246 (2001). doi:10.1046/j.1365-8711.2001.04022.x

41. R. L. Kurucz, Atlas: A Computer Program for Calculating Model Stellar Atmospheres, Smithsonian Astrophysical Observatory Special Report 309 (1970); http://kurucz.harvard.edu/papers/sa0309/saospecialreport309.pdf.

42. L. Sbordone, P. Bonifacio, F. Castelli, R. L. Kurucz, Mem. Soc. Astron. Ital. 5 (suppl.), 93 (2004).

43. R. L. Kurucz, http://kurucz.harvard.edu.

44. R. L. Kurucz, E. H. Avrett, Solar Spectrum Synthesis. I. A Sample Atlas from 224 to $300 \mathrm{~nm}$, Smithsonian Astrophysical Observatory Special Report 391 (1981); http://kurucz.harvard.edu/papers/sao391/saosr391.pdf.

45. A. Dotter, B. Chaboyer, D. Jevremović, E. Baron, J. W. Ferguson, A. Sarajedini, J. Anderson, The ACS Survey of Galactic Globular Clusters. II. Stellar Evolution Tracks, Isochrones, Luminosity Functions, and Synthetic Horizontal-Branch Models. Astron. J. 134, 376-390 (2007). doi:10.1086/517915

46. A. Dotter, MESA isochrones and stellar tracks (MIST) 0: Methods for the construction of stellar isochrones. Astrophys. J. Suppl. Ser. 222, 8 (2016). doi:10.3847/0067-0049/222/1/8

47. M. Salaris, A. Chieffi, O. Straniero, The $\alpha$-enhanced isochrones and their impact on the FITS to the Galactic globular cluster system. Astrophys. J. 414, 580 (1993). doi:10.1086/173105

48. A. Pietrinferni, S. Cassisi, M. Salaris, F. Castelli, A Large Stellar Evolution Database for Population Synthesis Studies. II. Stellar Models and Isochrones for an $\alpha$-enhanced Metal Distribution. Astrophys. J. 642, 797-812 (2006). doi:10.1086/501344

49. A. N. Cox, Allen's Astrophysical Quantities (AlP Press, ed. 4, 2000).

50. R. L. Kurucz, Mem. Soc. Astron. Ital. 8 (suppl.), 14 (2005).

51. A. McWilliam, R. A. Bernstein, Globular Cluster Abundances from High-Resolution Integrated-Light Spectra. I. 47 Tuc. Astrophys. J. 684, 326-347 (2008). doi:10.1086/589957

52. P. Eitner, M. Bergemann, S. S. Larsen, NLTE modelling of integrated light spectra. Astron. Astrophys. 627, A40 (2019). doi:10.1051/0004$6361 / 201935416$

53. F. Castelli, Mem. Soc. Astron. Ital. 8 (suppl.), 44 (2005). 
54. HIRES Home Page, https://www2.keck.hawaii.edu/inst/hires/.

55. I. King, The structure of star clusters. I. An empirical density law. Astron. J. 67, 471 (1962). doi:10.1086/108756

56. K. Z. Stanek, P. M. Garnavich, Distance to M31 with the Hubble Space Telescope and Hipparcos Red Clump Stars. Astrophys. J. 503, L131-L134 (1998). doi:10.1086/311539

57. S. S. Larsen, J. P. Brodie, A. Sarajedini, J. P. Huchra, Structural Parameters and Dynamical Masses for Globular Clusters in M33. Astron. J. 124, 2615-2624

(2002). doi:10.1086/344110

\section{ACKNOWLEDGMENTS}

We thank Mark Gieles and Else Starkenburg for helpful discussions and comments on the manuscript, and Don VandenBerg for valuable advice on the selection of isochrones. Comments from the anonymous referees helped improve the presentation. The data were obtained at the W. M. Keck Observatory, which is operated as a scientific partnership among the California Institute of Technology, the University of California and the National Aeronautics and Space Administration. The Observatory was made possible by the generous financial support of the W. M. Keck Foundation. The authors wish to recognize and acknowledge the very significant cultural role and reverence that the summit of Maunakea has always had within the indigenous Hawaiian community. We are most fortunate to have the opportunity to conduct observations from this mountain. Funding: AJR was supported by National Science Foundation grant AST-1616710, and as a Research Corporation for Science Advancement Cottrell Scholar. JPB acknowledges support from HST grant HST-GO15078. Author contributions: JPB secured the observing time for this project, all authors contributed to the planning of the observations, and the inclusion of EXT8 as a target was suggested by AJR. AW conducted the observations. SSL carried out the data reduction and analysis and drafted the paper. All authors assisted in the interpretation of the results and writing of the paper. Competing interests: None. Data and materials availability: The average measured abundances are listed in table S2 and individual measurements are in tables S3 to S9. The raw observations of EXT8 and Hodge III are available in the W. M. Keck Observatory Archive at http://koa.ipac.caltech.edu, EXT8 under program ID U177Hr, Semester 2019B, and Hodge III under program ID U040Hr, Semester 2015B, P.I. Brodie. The UVES observations of M15 are available in the ESO archive at http://archive.eso.org, program ID 095.B-0677(A), P. I. Larsen. The CFHT MegaCam images of EXT8 are available in the CFHT data archive at http://www.cadc-ccda.hia-iha.nrc-cnrc.gc.ca/en/cfht, proposal ID 16BC25, product IDs 2011389p, 2011390p, and 2011391p. The spectral analysis was carried out with the ISPy3 code

https://github.com/soerenslarsen/ISPy3 (37), and the King model fitting was done with the Baolab code (38), available at https://github.com/soerenslarsen/baolab, DOI 10.5281/zenodo.4036106.

\section{SUPPLEMENTARY MATERIALS}

science.sciencemag.org/cgi/content/full/science.abb1970/DC1

Materials and Methods

Supplementary Text

Figs. S1 to S3

Tables S1 to S10

References (39-57)

7 February 2020; accepted 30 September 2020

Published online 15 October 2020

10.1126/science.abb1970 


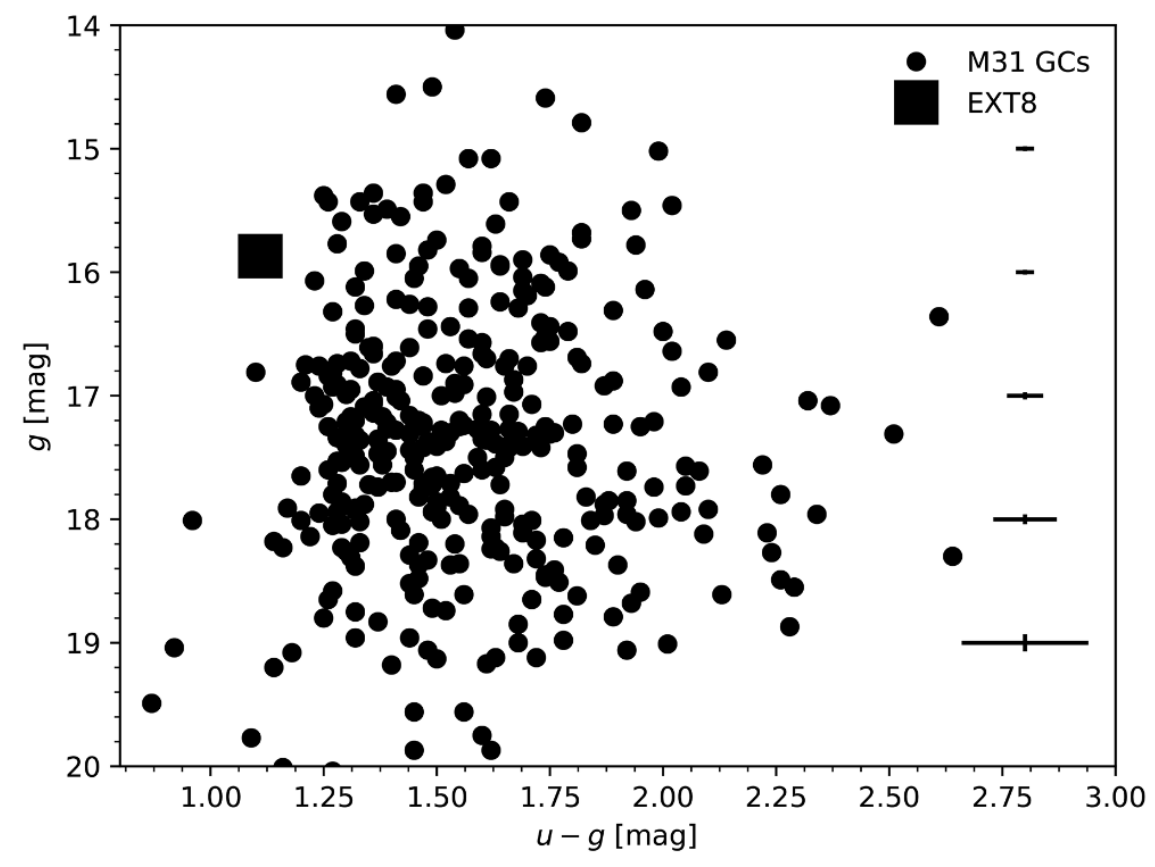

Fig. 1. Color-magnitude diagram for globular clusters in M31 (11). No correction for dust reddening has been applied. EXT8 is marked with a large square, and has one of the bluest $u-g$ colors among the GCs in M31. Typical one-sigma error bars are shown on the right. 


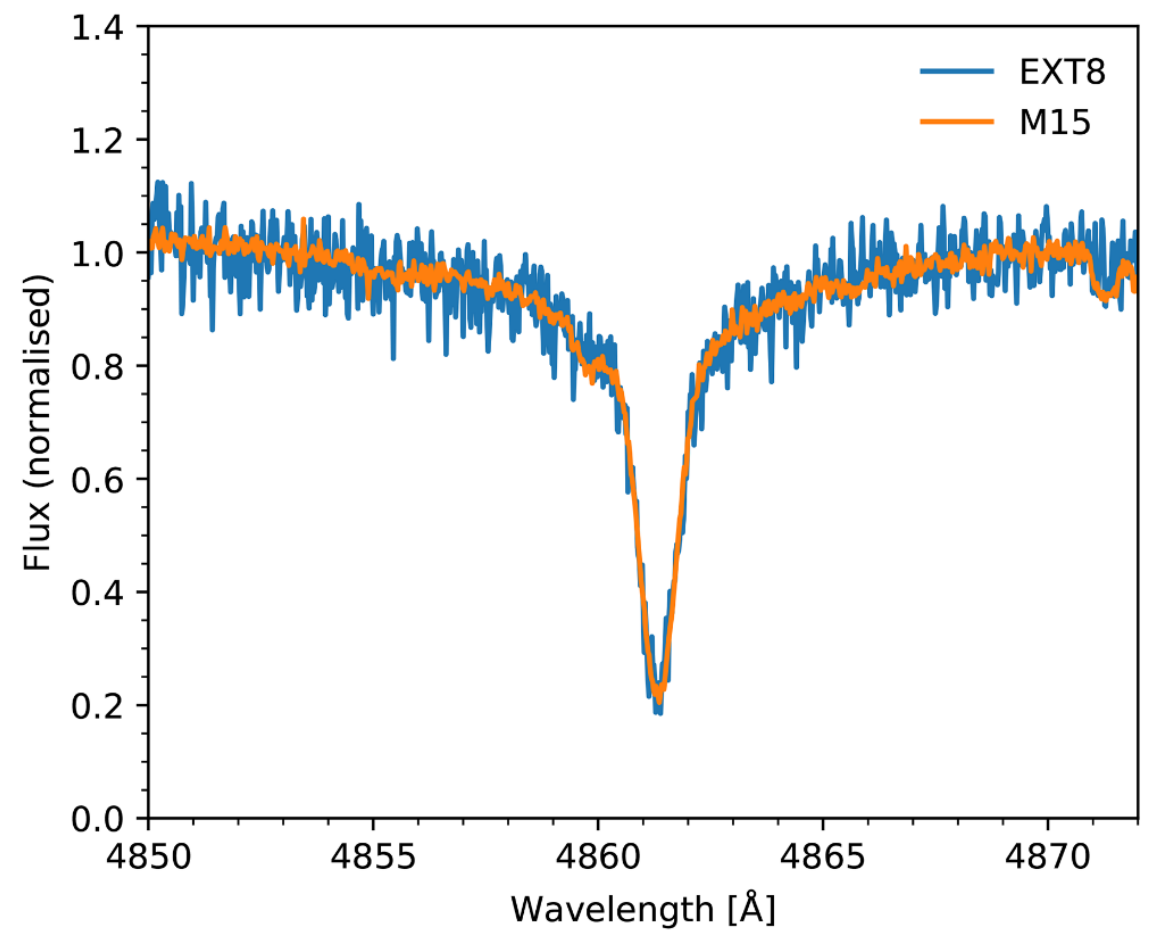

Fig. 2. Comparison of the $H \beta$ lines in the spectra of EXT8 (blue) and Messier 15 (orange). The very similar $\mathrm{H} \beta$ line profiles in the two spectra indicate similar, old ages for the two clusters. 

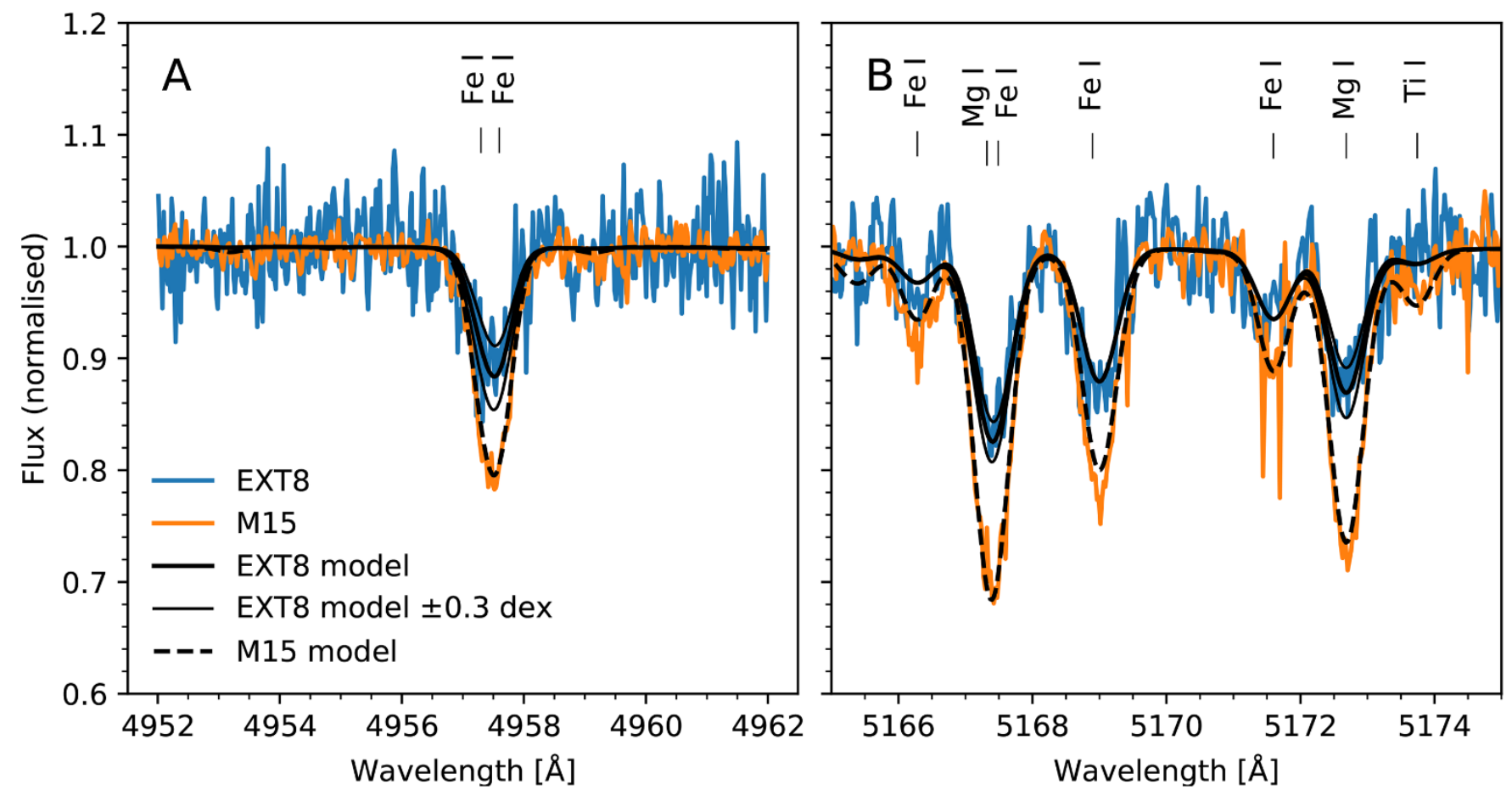

Fig. 3. Iron and magnesium features in the spectra of EXT8 (blue) and Messier 15 (orange). The best-fitting models are shown with thick black lines (for EXT8) and dashed black lines (for M15). Models for EXT8 in which the abundances have been varied by \pm 0.3 dex for iron $(A)$ and magnesium $(B)$ are shown with thinner lines. 


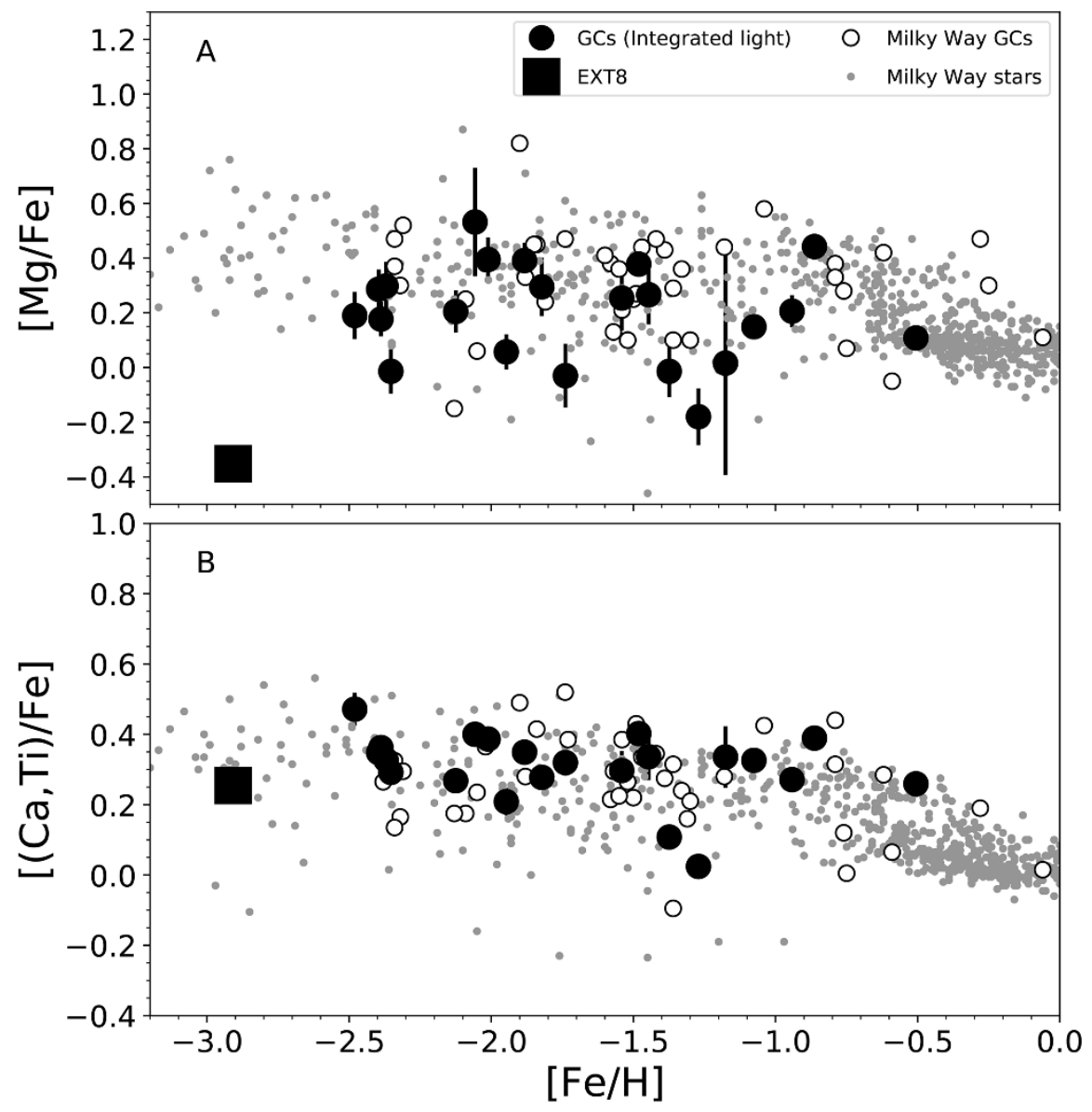

Fig. 4. Abundance measurements for EXT8 and other Galactic and extragalactic GCs. (A) $[\mathrm{Mg} / \mathrm{Fe}]$ as a function of $[\mathrm{Fe} / \mathrm{H}]$. (B) The average of $[\mathrm{Ca} / \mathrm{Fe}]$ and $[\mathrm{Ti} / \mathrm{Fe}]$. The large square shows our measurements for EXT8 while the filled circles show integrated-light measurements for GCs in the NGC 147, NGC 6822, Fornax, and WLM dwarf galaxies, M 33, and the Milky Way $(16,18)$. Data for resolved Milky Way GCs are shown with open circles (30) and data for field stars (31) with small gray dots. Error bars indicate the one sigma uncertainties. 